\title{
MULTICRITERIA DECISION-MAKING WEIGHTS AND A COMPETITIVE PRODUCT DESIGN
}

\section{Filip Tošenovský}

\section{Introduction}

Competitive environments exist or can be created for a majority of human activities. This is true because human activity can usually be performed by more than one subject, and to incite subjects to being competitive, it suffices to compare results of their activity on the basis of selected criteria or features which characterize these results. When competition occurs, one may react to it in different ways. Trying to distinguish oneself from others is one reaction, striving to catch up with the competition or overtake it might be another reaction. In economy, where economic survival and prosperity is often the ultimate objective, it might be necessary to overtake, or at least catch up with, the competition, as being only different may not be perceived as a sufficient guarantee of future financial stability. Staying in economy, where human activity results in a product, and holding to the idea that it is advisable to have at least as good a product as the competition, two questions arise:

1. How to compare two typologically identical products in a competitive environment?

2. If a product is worse than another, how to change its features to make it competitive?

Regarding the first question, it is not a problem to compare two products of the same type, as long as they are compared by a single criterion. The product which is better by that criterion is simply better overall. More often, however, products are compared by more criteria, which makes it more complicated to make a judgement which product is better, especially when one product is better than another by some criteria and worse at the same time by other criteria. In the less favourable case, it is possible to assign an aggregate value to each product so that the products are eventually comparable. There are various approaches how to determine such an aggregate value. One of the approaches assigns nonnegative weights $w_{i}, i=1,2, \ldots, n$, the sum of which equals one, to $n$ criteria we work with when evaluating products, and the product aggregate value is then given by the weighted average $h_{1} w_{1}+h_{2} w_{2}+\ldots+h_{n} w_{n}$, where $h_{i}$ is an evaluation of the $i$-th feature of the product, the feature being defined by one of the criteria [5]. The features are actually criteria defined by the customer. The evaluations $h_{i}$ 's are given or designed by the product maker. The features discussed may include such characteristics as product price, product power efficiency, product battery life, and so on. If a feature can be looked at in more than one way (product colour, for instance, can be discussed in terms of its aesthetic value or its potentially adverse effect on human health), then each of these interpretations may represent a separate feature (in the case of colour, both its aesthetic value and its safety would represent two separate product characteristics). The strength of the weighted-average approach is clear: in a group of typologically identical products, each product is evaluated with a single number representing an aggregation of the product information. Since there is only one number that is assigned to each product, the products can be arranged from best to worst. Although not the only one, this approach is natural and reflects importance of all the criteria considered. This type of aggregate product evaluation will be utilized in this paper, and will be considered a model that describes, at least approximately, the decisionmaking process customers go through when buying a product.

As for the second question, assuming that a lower aggregate product value, as described above, means a worse product, the second question asks us how to adjust the feature levels $h_{i}$ 's of a product so that its new 
aggregate value, implied by the adjusted $h_{i}$ 's, is higher than its existing aggregate value. To answer this question, the weights $w_{i}$ 's must be known because otherwise it is impossible to calculate either the original or the adjusted aggregate value of the product. But how to set the weights if they are defined by the customer? It is the customer who knows which of the criteria is important and which isn't. Product manufacturers, who would like to adjust the evaluations $h_{i}$ 's to become more competitive, do not know the weights, and thus cannot know how to change their product characteristics accordingly.

This paper focuses on answering the second question, utilizing the model of the product evaluation described above. The paper proposes an approach which results in finding a specific set of weights without knowing much about the customer. The approach benefits from the general theory of optimization. After analysing the problem of weights, the paper completes the description of the procedure for finding the weights with a cost-effective way of improving the evaluations $h_{i}$ 's so that the aggregate value of the upgraded product was on par with the competition as cheaply as possible. Further, regarding the weights, the procedure presented in the paper is compared with another approach that finds alternative weights. This alternative approach offers itself as an answer to the second question more naturally than the approach proposed in the paper, but, in fact, it will turn out to be a less favourable approach, since it may often not have a realistic interpretation. Also, the proposed procedure always leads to a unique result, as will be shown, and this cannot be said about the alternative procedure. The paper contains examples of the theoretical results, as well. The examples support the validity of the theory proved in the paper.

Generally speaking, the procedure we are about to deal with technically lies in optimizing a mathematical expression - in minimizing the expression. The minimization will seek weights which will bring an assessment of one product (a worse one) to an assessment of another product (a better one) as close as possible. The decision to minimize the difference in assesments of two products is based on the effort to utilize the minimization in capturing market dynamics, or its change, in the following sense of the word: let us imagine that we are a producer who prospers more than our competitor in terms of product sales, the competitor serving for our business purposes as a certain standard. Let us assume that the competitor starts to overtake us in the product sales. Such a move necessarily suggests the market has changed - our product starts to be percieved differently by customers. We are interested in how such a change might look like, according to a model, so that we could respond to the change accordingly. In trying to find out the new form of the customer perception of our product, we could base our inference about the customer on the fact that the customer evaluates the product somehow, and we can try to model this assessment in a "natural" way, and on the fact that, at the moment, we do not fall behind the competitor too much with our product. We will never find out how much we fall behind the competitor with our product, described by its features and their importance, nor the customer is likely to describe it exactly due to the amount of vagueness related to weights. However, we may approximate the currently small difference in the perception of our and the competitive product with the minimal difference in this perception. None the less, more mathematical expressions offer themselves as a description of the difference in the product perception which could be minimized. We will show, as we already outlined in the previous paragraph, that some of these expressions or approaches serve our purposes better than others. The minimization can be used as a model that helps us discover a potential distortion in our expectation of what the customer wants from our product. Maybe, the customer began to want something else than what we had expected. Of course, this change will also be reflected, among other things, in our product sales. If the model is to function reasonably, it should not be used with too much of a delay after it begins to be obvious that our market position stopped being superior to that of our competitor.

Before presenting the approach or the model, let us note that different procedures to set weights were proposed in the past by many authors, using various mathematical tools. As early as the 1970s, Pekelman and Sen [11] exploited the possibility of modelling customers' behaviour with the mathematical programming optimization, although their procedures were rather limited to specific market segments, particularly to cereal products. Most scientific 
papers devoted to this subject dealt with weights which are defined by decision makers themselves who know best their subjective approaches to decision problems. This is not our situation, however. Our situation is such that the mind of decision makers/customers remains unknown to those who want to sell their products. To give some typical examples of the papers devoted to weights defined by decision makers themselves, Nutt [8], for instance, compared several ways of determining the weights by decision makers. Choo and Wedley [2] used the linear programming methodology and decision makers' past rulings to find weights which would lead to multicriteria problem solutions complying with the decision makers' notion of how the solution should look like. Solymosi and Dombi [12] worked out a way of setting weights, which required information from decision makers in the form of inequalities. From more recent papers, Choo, Schoner and Wedley [3] were preoccupied with pitfalls of decision-making weights interpretation, giving recommendations to decision makers how to proceed when defining weights. A step towards working with weights without prior information appeared in a hybrid approach introduced by $\mathrm{Ma}$, Fan and Huang [7]. The approach combines objective information and subjective knowledge to determine weights; the procedure still requires some specific knowledge of the decision maker's behaviour. Most recently, the scientific progress in the field of multicriteria decision-making has focused on modelling the uncertainty embedded in the lack of information with fuzzy sets and numbers. These approaches either require all or some information on the weights. Wang, Li and Wang [15] presented such approach, their procedures requiring some information from decision makers in the form of inequalities. Luo et al. [6] presented an approach with known weight information, the approach being based on weighted correlation coefficients in an interval-valued intuitionistic fuzzy environment. A similar, but improved approach applied to group decision-making was proposed by Park et al. [10]. The approach requires some information on attribute weights to be known. Finally, a fuzzy approach based on an interval-valued intuitionistic fuzzy decision matrix, was introduced by Ye [16].

As can be seen, the research has been centred largely on methods which require at least some information on criteria weights.
Some methods have been presented, which are very general and do not require much information on weights, as is the case of Ye [16]. However, this method, for instance, suggests that a special fuzzy decision matrix is available from the decision maker who solves the problem. In our case, it is hard to imagine that a customer will have a good enough command of the fuzzy set theory to be able to provide the product maker with such an input matrix. In light of that, our approach, as will be seen, is simpler, and doesn't require any specific prior information on the weights from the customer; it rather draws on general information about his behaviour which is reflected in the product sales.

\section{Weights}

In this section, we derive a set of weights which the customer might potentially use to assess the importance of individual product features, or decision criteria, and judge on the aggregate value of the entire product, using the weighted average technique. We derive the set of weights generally and explicitly, drawing on matrix properties and optimization techniques. Technical details concerning the matrix properties are contained in the appendix to this paper. Throughout the text, the following is assumed to hold true for the subsequent procedures to be correct:

1. We improve a product with an aggregate value $b_{1} w_{1}+b_{2} w_{2}+\ldots+b_{n} w_{n}$, where $b_{i}$ is a positive evaluation of the $i$-th quantitative product feature. This prerequisite automatically assumes that it is possible to calculate the aggregate value in the first place, i.e. no different physical units are considered here. Each evaluation, which may result from a test performed for customers by a scholarly journal using an evaluation scale without physical units, is in fact determined by product makers in their production process. The symbol $w_{i}$ represents the $i$-th weight defined for the $i$-th criterion or feature by the customer. We shall often refer to this product as our product in the paper. Our product is compared to a competitive product valued at $c_{1} w_{1}+c_{2} w_{2}+\ldots+c_{n} w_{n}$, where $c_{i}$ is a positive evaluation of the $i$-th quantitative feature of the competitive product. The same set of features or criteria and weights is used for both products. 
2. A higher value of the $i$-th product feature means a better product feature.

3. $b_{i}<c_{i}, i=1,2, \ldots, n$, that is $\sum_{k} b_{k} w_{k}<\sum_{k} c_{k} w_{k}$. It must be stressed that this condition may not be as strict as it seems at a glance. If a product feature violates the condition, which actually means that our product is better by that feature than the competitor's product, it probably won't be something that matters much to the customer given the fact that we still experience lower product sales. It may then make sense to leave out this characteristic from further analysis since it is not relevant, and the condition 3) will be met without much harm done to the entire modelling. We can even get an idea whether such a feature, say the $i$-th feature, is important or not by solving the following simple linear problem: maximize $w_{i}$ subject to $\sum_{k} w_{k}=1$ and $\sum_{k} b_{k} w_{k} \leq \sum_{k} c_{k} w_{k}$. If the maximized value of $w_{i}$ is smaller than a predetermined number, say 0.1 , we may abolish this feature.

4. We work with a real Euclidean metric space, whenever metric properties occur in the text [9].

5. We assume that we are in a market where customers percieve the product consistently, i.e. the weights assigned to the product features are similar across the customers. If a product feature is important to a selected customer, the importance being expressed by the corresponding weight, that feature is also important to a similar extent to most other customers. An analogy holds true for less important product features. If this prerequisite was too far removed from reality, there would not be much sense in dealing with the techniques we are about to describe, as each customer would take a severely different approach to the product characteristics. In this case, it would not make sense to model the weights assigned to the product by the whole market because such a model would probably be too rough. The prerequisite will definitely be more easily met in an electronics market, where certain trends are fairly obvious, regarding what customers like and dislike about a product, rather than in a highly subjective market, such as the one with paintings and other works of art, for instance. No real trends in product perception exist in the latter market.

Given the assumptions, let us now return to the problem of weights. Finding weights, as proposed in this paper, benefits from the following property: if $a_{i}, i=1,2, \ldots, k$, $k>1$, are positive numbers, the symmetric $(k-1) \times(k-1)$ matrix

$$
\left(\begin{array}{cccc}
a_{1}+a_{k} & a_{k} & \cdots & a_{k} \\
a_{k} & a_{2}+a_{k} & \cdots & a_{k} \\
\cdots & \cdots & \cdots & \cdots \\
a_{k} & a_{k} & \cdots & a_{k-1}+a_{k}
\end{array}\right)
$$

is positive definite, and thus has an inverse, as well. A proof of this statement is in the appendix.

We shall use the matrix property just described to find the weights. First, let us look back at the aggregate value of our product, given by the expression $b_{1} w_{1}+b_{2} w_{2}+\ldots+b_{n} w_{n}$. We could find weights $w_{i}, i=1,2, \ldots, n$, which solve the optimization problem

$$
\min _{\mathbf{w}=\left[w_{1}, w_{2}, \ldots, w_{n}\right] \in S}\left(\sum_{i} b_{i} w_{i}-\sum_{i} c_{i} w_{i}\right)^{2}
$$

where

$$
S=\left\{\mathbf{w} \in R^{n}: \sum_{i=1}^{n} w_{i}=1, w_{i} \geq 0, i=1,2, \ldots, n\right\} .
$$

This approach is very tolerant of the extent to which our product falls behind the competitive product. This approach implicitly assumes that our product falls behind the competitive product as little as possible in the sense given by (2). It is an optimistic outlook on how the customer assesses the inferior product. The customer prefers a different product, the competitive one, to our product that we want to improve, but only a little. The difference in the product evaluation is modelled as minimal. This procedure seems very natural, if one is an optimist, but the opposite will later turn out to be true - this procedure is not very natural. We are going to propose another optimistic concept. defining

$$
a_{i}=\left(c_{i}-b_{i}\right)^{2}, i=1,2, \ldots, n
$$

and using for convenience the notation

$$
\mathbf{w}=\left(w_{1}, w_{2}, \ldots, w_{n}\right),
$$


we are looking for weights which instead solve the following problem

$$
\operatorname{minimize} f(\mathbf{w})=\sum_{i=1}^{n} a_{i} w_{i}^{2} \text { on } S \text {. }
$$

The concept given by (6) is different. Looking at the overall value of our product given by $b_{1} w_{1}+b_{2} w_{2}+\ldots+b_{n} w_{n}$, we see that each term in this summation can be viewed as a contribution to the overall value of the product. Let us define a vector of contributions $\tilde{\mathbf{b}}=\left(b_{1} w_{1}, b_{2} w_{2}, \ldots, b_{n} w_{n}\right)$ for our product and a vector of contributions $\tilde{\mathbf{c}}=\left(c_{1} w_{1}, c_{2} w_{2}, \ldots, c_{n} w_{n}\right)$ for the competitive product. Doing so, we optimize the function on the same set as before, but this time the optimization or minimization concerns the expression $\|\tilde{\mathbf{c}}-\tilde{\mathbf{b}}\|^{2}$, where the norm is Euclidean, as assumed. Therefore, we actually minimize an Euclidean distance between two vectors of contributions to the overall product value.

Let us solve the optimization problem given by (6). The coefficients $a_{i}$ 's are positive by assumption 3 . The set $S$ is clearly bounded, since each weight is smaller or equal to 1 and nonnegative. The set $S$ is also closed. To prove this, one needs to show that any point $\mathbf{w} \in R^{n}$ whose distance from $S$ is zero belongs to $S$ [9]. Given a point $\mathbf{w} \in R^{n}$ at a zero distance from $S$, a sequence $\left\{\mathbf{w}_{k}\right\}_{k=1}^{\infty}$ of points $\mathbf{w}_{k}$ from $S$ exists such that $\lim \mathbf{w}_{k} \stackrel{k=1}{=} \mathbf{w}$ [9]. Thus, for the function $h(\mathbf{w})=\sum_{i=1}^{k \vec{n}^{\infty}} w_{i}-1, \quad h(\mathbf{w})=\lim _{k \rightarrow \infty} h\left(\mathbf{w}_{k}\right)=0$, since the function is continuous. Also, $\mathbf{w}_{k} \geq \mathbf{0}$ implies $\mathbf{w} \geq \mathbf{0}$. Therefore, $\mathbf{w} \in S$.

Since the function to be minimized is continuous everywhere and $S$ is closed and bounded, the optimization problem (6) has a solution. We will show that this solution is unique: It is obvious that (6) is a convex problem, and so, to find its global solution, it suffices to find its local solution. We will find a local solution, and since the function in (6) is strictly convex everywhere, the local solution will define the unique global solution. To solve

$$
\min _{\mathbf{w} \in S} f(\mathbf{w}),
$$

we shall, however, solve a simpler problem

$$
\min _{\mathbf{w} \in S^{*}} f(\mathbf{w}),
$$

where

$$
S^{*}=\left\{\mathbf{w} \in R^{n}: \sum_{i=1}^{n} w_{i}=1\right\} .
$$

As we shall see, the solution $\mathbf{w}^{*}=\underset{\mathbf{w} \in S^{*}}{\arg \min } f(\mathbf{w}) \quad$ satisfies $\quad \mathbf{w}^{*} \in S \subseteq S^{*}$. Since $\mathbf{w}^{*}$ will solve the problem on a "larger" set, and belongs to $S$, it will also solve the original problem. Let us solve the alternative problem given by (8) and (9) by finding its unique local minimum. To do so, we use the substitution $1-\sum_{i=1}^{n-1} w_{i}=w_{n}$. As is known, the substitution converts the constrained problem to an unconstrained one. Inserting the substitution in $f(\mathbf{w})$, this function is transformed into

$$
g\left(w_{1}, w_{2}, \ldots, w_{n-1}\right)=\sum_{i=1}^{n-1} a_{i} w_{i}^{2}+a_{n}\left(1-\sum_{i=1}^{n-1} w_{i}\right)^{2}
$$

If a local minimum of $g$ is achieved at a point $\mathbf{w}^{*}=\left(w_{1}^{*}, w_{2}^{*}, \ldots, w_{n-1}^{*}\right)$, the set of equations

$$
\partial g\left(\mathbf{w}^{*}\right) / \partial w_{i}=0, i=1,2, \ldots, n-1
$$

must hold, or

$$
\left(a_{j}+a_{n}\right) w_{j}^{*}+a_{n} \cdot \sum_{k=1, k \neq j}^{n-1} w_{k}^{*}=a_{n}, j=1,2, \ldots, n-1 .
$$

In matrix notation, we work with equations

$$
A \cdot\left(\begin{array}{c}
w_{1}^{*} \\
w_{2}^{*} \\
\vdots \\
w_{n-1}^{*}
\end{array}\right)=\left(\begin{array}{c}
a_{n} \\
a_{n} \\
\vdots \\
a_{n}
\end{array}\right),
$$

where $A$ is the matrix (1). Since the matrix has its inverse (see the appendix),

$$
\left(\begin{array}{c}
w_{1}^{*} \\
w_{2} \\
\vdots \\
w_{n-1}^{*}
\end{array}\right)=A^{-1} \cdot\left(\begin{array}{c}
a_{n} \\
a_{n} \\
\vdots \\
a_{n}
\end{array}\right)
$$

is the only solution to equations (13). Further, since the Hessian matrix

$$
H=\left(h_{i j}=\partial^{2} g\left(\mathbf{w}^{*}\right) / \partial w_{i} \partial w_{j}\right)=2 A
$$


is positive definite, $\left(w_{1}^{*}, w_{2}^{*}, \ldots, w_{n-1}^{*}\right)$ is the point where $g$ attains its strict local minimum, and thus

$$
\mathbf{w}^{*}=\underset{\mathbf{w} \in S^{*}}{\arg \min } f(\mathbf{w})=\left(w_{1}^{*}, w_{2}^{*}, \ldots, w_{n-1}^{*}, w_{n}^{*}\right),
$$

where

$$
w_{n}^{*}=1-\sum_{k=1}^{n-1} w_{k}^{*} .
$$

Selecting any one j-th equation in (12), we see that there exists an index $l \in\{1,2, \ldots, n-1\}$ such that $w_{l}^{*}>0$. In the opposite case, if each $w_{j}^{*}, j=1,2, \ldots, n-1$, was nonpositive, the $j$-th equation (18) could not hold, since its right-hand side is positive. The $l$-th equation, $1 \leq l \leq n-1$,

$$
a_{l} w_{l}^{*}=a_{n} w_{n}^{*}
$$

then implies that $w_{n}^{*}$ is positive, and thus also each $w_{j}^{*}=\left(a_{n} / a_{j}\right) w_{n}^{*}, j=1,2, \ldots, n-1$, is positive. In other words, $\mathbf{w}^{*} \in S$, and $\mathbf{w}^{*}$ therefore solves the original problem $\min f(\mathbf{w})$. It is also the only solution to the originâf ${ }^{\mathcal{E}}$ problem because $f(\mathbf{w})$ is strictly convex everywhere.

Returning to our product, the customer evaluates the product, according to our concept, by the number $\mathbf{w}^{*} \mathbf{b}^{\prime}$, where $\mathbf{w}^{*}$ satisfies (14) and (17) and $\mathbf{b}=\left(b_{1}, b_{2}, \ldots, b_{n}\right)$ is the vector of individual evaluations of the product features. The competitive product is valued at $\mathbf{w}^{*} \mathbf{c}^{\prime}$ by the customer, where $\mathbf{c}=\left(c_{1}, c_{2}, \ldots, c_{n}\right)$ is the vector of individual evaluations of the competitive product features. We have $\mathbf{w}^{*} \mathbf{b}^{\prime}<\mathbf{w}^{*} \mathbf{c}^{\prime}$ by assumption 3 . Our next objective is to catch up with the competition in a cost-effective way, as was outlined at the beginning of the paper. To be more precise, we are looking for a new vector of evaluations $\mathbf{c}_{\text {new }}=\left(c_{\text {new }, 1}, c_{\text {new }, 2}, \ldots, c_{\text {new }, k}\right)$ of our product features, for which $\mathbf{w}^{*} \mathbf{c}^{\prime} \leq \mathbf{W}^{\prime} \mathbf{c}_{\text {new }}^{\prime}$. Assuming that we want to achieve this objective without degrading the current parameters of our product, i.e. $\mathbf{c}_{\text {new }} \geq \mathbf{b}$ is required, the change of our product features will cost $\mathbf{P}\left(\mathbf{c}_{\text {new }}-\mathbf{b}\right)^{\prime}$, where $\mathbf{P}=\left(P_{1}, P_{2}, \ldots, P_{n}\right)$ is the vector of prices. Each price contained in $\mathbf{P}$ represents expenses on the unit improvement of the corresponding product feature. If we want to minimize these costs, which is what would mean cost-effective in our concept, we additionally need to solve the linear program

$$
\min _{\mathbf{c}_{\text {new }}} \mathbf{P}\left(\mathbf{c}_{\text {new }}-\mathbf{b}\right)^{\prime}
$$

subject to

$$
\mathbf{w}^{*} \mathbf{c}_{\text {new }}^{\prime} \geq \mathbf{w}^{*} \mathbf{c}^{\prime}, \mathbf{c}_{\text {new }} \geq \mathbf{b}
$$

\section{Alternative Approach}

As mentioned earlier, weights that minimize the expression $\|\mathbf{c}-\mathbf{b}\|^{2}$, where $\mathbf{b}=\left(b_{1} w_{1}, b_{2} w_{2}, \ldots, b_{n} w_{n}\right)$ and $\mathbf{c}=\left(c_{1} w_{1}, c_{2} w_{2}, \ldots, c_{n} w_{n}\right)$, are found. From the mathematical point of view, however, it might seem more natural to seek the weights that solve the problem

$$
\min _{\mathbf{w} \in S} h(\mathbf{w}),
$$

where

$$
h(\mathbf{w})=h\left(w_{1}, w_{2}, \ldots, w_{n}\right)=\left(\sum_{i=1}^{n} c_{i} w_{i}-\sum_{i=1}^{n} b_{i} w_{i}\right)^{2} .
$$

It is another optimistic approach to finding the weights, since this approach assumes that the aggregate value of our product falls behind that of the competitor, but not much, and the amount "not much" is modelled by (21). This procedure, however, presents complications if it is compared to the earlier procedure. When finding the weights, we preferred a solution which would be unique. Although not explicitly said, we also preferred a solution in which all product features were considered, if possible, i.e. their weights were positive. Otherwise, it doesn't make much sense to work with all the criteria. The solution to the optimization problem (21), however, does not often meet these requirements, and thus renders itself a less favourable procedure. To see these drawbacks, let us first consider a case when $c_{i}-b_{i}=k$ for $i=1,2, \ldots, n$, where $k$ is a constant. Then

$$
\begin{aligned}
& \left(\sum_{i=1}^{n} c_{i} w_{i}-\sum_{i=1}^{n} b_{i} w_{i}\right)^{2}=k^{2} \sum_{i=1}^{n} \sum_{j=1}^{n} w_{i} w_{j}= \\
& =k^{2} \sum_{i=1}^{n}\left(\sum_{j=1}^{n-1} w_{i} w_{j}+w_{i}\left(1-\sum_{j=1}^{n-1} w_{j}\right)\right)=k^{2} \sum_{i=1}^{n} w_{i}=k^{2}
\end{aligned}
$$

on $S^{*}$. 
Thus, $h\left(w_{1}, w_{2}, \ldots, w_{n}\right)$ being constant on $S \subset S^{*}$, the problem (21) has no unique solution. Further, setting first $n=2$ for simplicity, and having by assumption $k_{1}=c_{1}-b_{1}>0, k_{2}=c_{2}-b_{2}>0, k_{1} \neq k_{2}$, the function $g\left(w_{1}\right)=h\left(w_{1}, 1-w_{1}\right)$ satisfies

$$
g^{\prime}\left(w_{1}\right)=2 w_{1}\left(k_{1}-k_{2}\right)^{2}+2 k_{2}\left(k_{1}-k_{2}\right),
$$

which is certainly positive for $k_{1}>k_{2}$ and $w_{1} \geq 0$, or negative for $k_{1}<k_{2}$ and $w_{1} \in[0,1]$. Therefore, there cannot be a minimum of $h\left(w_{1}, w_{2}\right)$ on $S$ such that $0<w_{1}<1,0<w_{2}<1$, because if there was, it would be a local minimum of $g\left(w_{1}\right)=h\left(w_{1}, 1-w_{1}\right)$, and the derivative $g^{\prime}\left(w_{1}\right)$ would be zero. The only point from $S$ in which $h\left(w_{1}, w_{2}\right)$ can attain its minimum is therefore at a vertex of $S$, or at an extreme point of $S$. In the more general case, let us write

$$
\begin{aligned}
& h\left(w_{1}, w_{2}, \ldots, w_{n}\right)=\left(\sum_{i=1}^{n} c_{i} w_{i}-\sum_{i=1}^{n} b_{i} w_{i}\right)^{2}=\left(\sum_{i=1}^{n} k_{i} w_{i}\right)^{2}= \\
& =\left(\sum_{i=1}^{n-1} k_{i} w_{i}+k_{n}\left(1-\sum_{i=1}^{n-1} w_{i}\right)\right)^{2}=g\left(w_{1}, w_{2}, \ldots, w_{n-1}\right) .
\end{aligned}
$$

\section{We have}

$$
\begin{aligned}
& \frac{\partial g\left(w_{1}, w_{2}, \ldots, w_{n-1}\right)}{\partial w_{l}}=2\left(\left(k_{1}-k_{n}\right) w_{1}+\left(k_{2}-k_{n}\right) w_{2}+\ldots+\right. \\
& \left.+\left(k_{n-1}-k_{n}\right) w_{n-1}+k_{n}\right)\left(k_{l}-k_{n}\right), 1 \leq l \leq n-1 .
\end{aligned}
$$

If we assume $k_{n}<k_{l}, 1 \leq l \leq n-1$, the set of equations $\partial g\left(w_{1}, w_{2}, \ldots, w_{n-1}\right) / \partial w_{l}=0,1 \leq l \leq n-1$, does not have a solution satisfying $w_{i} \geq 0,1 \leq i \leq n-1$, since the coefficients $k_{i}$ ' $s$ are assumed to be positive. We could have also used a different substitution than $w_{n}=1-\sum_{i=1}^{n-1} w_{i}$, however; $w_{1}=1-\sum_{i=2}^{n} w_{i}$, for instance, which would give us a function $g\left(w_{2}, w_{3}, \ldots, w_{n}\right)$ having derivatives

$\frac{\partial g\left(w_{2}, w_{3}, \ldots, w_{n}\right)}{\partial w_{l}}=2\left(\left(k_{2}-k_{1}\right) w_{2}+\left(k_{3}-k_{1}\right) w_{3}+\ldots+\right.$

$\left.+\left(k_{n}-k_{1}\right) w_{n}+k_{1}\right)\left(k_{l}-k_{1}\right), 2 \leq l \leq n$.

Again, assuming $k_{1}<k_{l}, 2 \leq l \leq n$, the set of equations $\partial g\left(w_{2}, w_{3}, \ldots, w_{n}\right) / \partial w_{l}=0,2 \leq l \leq n$, does not have a solution satisfying $w_{i} \geq 0,2 \leq i \leq n$. It follows from here that whenever there is a unique minimum among the numbers $k_{i}=c_{i}-b_{i}, i=1,2, \ldots, n$, the problem $\min h(\mathbf{w})$ on $S$ does not have a solution in the interior of $S$. If it had, the point would be a local minimum of $h(\mathbf{w})$ not only on $S$, but also on $S^{*} \supset S$. This would mean, however, that the "projection" $g$, constructed from $h(\mathbf{w})$ through a suitable substitution, would have its derivatives equal to zero at that point. This possibility has just been ruled out for many cases, using various substitutions. If there is a solution to the problem $\min h(\mathbf{w})$ on $S$, it is not in the interior of $S$ in many cases, and so it must be on the boundary of $S$ in those cases, making itself an extreme solution. These cases are documented in the examples that follow.

We see that the procedure which seemed more natural is, on the contrary, more artificial. It does not always have a unique solution, and if it does have a solution, the solution is in many cases too extreme to be a good model of customers' preferences - it does not take into account all the criteria.

\section{Examples}

What follows is a series of examples working with four different criteria. In the examples, we find the weights, using the approach proposed in the paper. We also use the Solver module of the Excel spreadsheet programme to see that the procedure is correct. The computer result serves as an empirical verification of the validity of the explicit and existing mathematical solution. The weights in the examples are also found by the alternative approach, using the Solver. The examples show that all the points presented in the paper hold: our explicit formulas for calculating the weights and the extreme or unnatural character of the solution provided in many cases by the alternative approach.

\subsection{Example 1}

Set $\mathbf{c}=(7,5,2,9), \mathbf{b}=(5.2,2.7,1.1,6.4)$, i.e. $\mathbf{a}=\left(a_{1}, a_{2}, a_{3}, a_{4}\right)=(3.24,5.29,0.81,6.76)$.

We have

$\left(\begin{array}{l}w_{1}^{*} \\ w_{2}^{*} \\ w_{3}^{*}\end{array}\right)=A^{-1} \cdot\left(\begin{array}{l}a_{4} \\ a_{4} \\ a_{4}\end{array}\right)=\left(\begin{array}{ccc}10 & 6.76 & 6.76 \\ 6.76 & 12.05 & 6.76 \\ 6.76 & 6.76 & 7.57\end{array}\right)^{-1} \cdot\left(\begin{array}{l}6.76 \\ 6.76 \\ 6.76\end{array}\right)=\left(\begin{array}{l}0.164156 \\ 0.100542 \\ 0.656624\end{array}\right)$

and $\quad w_{4}^{*}=1-w_{1}^{*}-w_{2}^{*}-w_{3}^{*}=0.078678$. These are also the values returned by the 
Solver. If the same product feature levels were used to find the weights with the alternative approach, the alternative approach would give the result $\left(\tilde{w}_{1}, \tilde{w}_{2}, \tilde{w}_{3}, \tilde{w}_{4}\right)=(0,0,1,0)$ according to the Solver.

To catch up with the competition, we are solving the problem $\min \mathbf{P}\left(\mathbf{c}_{\text {new }}-\mathbf{b}\right)^{\prime}$ subject to $\mathbf{w}^{*} \mathbf{c}_{\text {new }}^{\prime} \geq \mathbf{w}^{*} \mathbf{c}^{\prime}, \mathbf{c}_{\text {new }} \geq \mathbf{b}$, where the vector of expenses $\mathbf{P}$ is known and specified beforehand. Let us assume for our illustrative purposes that $\mathbf{P}=(50,30,40,45)$. Then the linear problem is optimized for the proposed weights by the vector $\mathbf{c}_{\text {new, optimal }}=(5.2,2.7,3.11,6.4)$, the result being obtained by the Solver, for instance, and the cost of the change amounts to 80.5 currency units.

\subsection{Example 2}

Setting the vectors of product features $\mathbf{c}=(3.56,8.12,7.81,4.35), \mathbf{b}=(2.18,7.15,6.13,3.03)$, i.e.

$\mathbf{a}=\left(a_{1}, a_{2}, a_{3}, a_{4}\right)=(1.9044,0.9409,2.8224,1.7424)$, gives the weights

$\left(\begin{array}{l}w_{1}^{*} \\ w_{2}^{*} \\ w_{3}^{*}\end{array}\right)=A^{-1} \cdot\left(\begin{array}{l}a_{4} \\ a_{4} \\ a_{4}\end{array}\right)=\left(\begin{array}{lll}3.6468 & 1.7424 & 1.7424 \\ 1.7424 & 2.6833 & 1.7424 \\ 1.7424 & 1.7424 & 4.5648\end{array}\right)^{-1} \cdot\left(\begin{array}{l}1.7424 \\ 1.7424 \\ 1.7424\end{array}\right)=\left(\begin{array}{l}0.208692 \\ 0.422398 \\ 0.140814\end{array}\right)$

and $\quad w_{4}^{*}=1-w_{1}^{*}-w_{2}^{*}-w_{3}^{*}=0.228096$. These are also the values returned by the Solver. If the same product individual evaluations were used to find the weights with the alternative approach, the alternative approach would give the result $\left(\tilde{w}_{1}, \tilde{w}_{2}, \tilde{w}_{3}, \tilde{w}_{4}\right)=(0,1,0,0)$ according to the Solver.

As earlier, we need to change the product features to $\mathbf{c}_{\text {new, optimal }}=(2.18,10.075,6.13,3.03)$ for 87.74 currency units if the proposed weights are used, and we want to stay competitive.

\section{Conclusions}

This paper dealt with the desire to establish product features that would lead to a competitive product. What is competitive and what is not is entirely defined by customers, therefore companies can only guess at this definition, or put another way, they can only model the customers' behaviour. A very natural model exists in the framework of multicriteria decisionmaking, which evaluates the whole product with a weighted average, the weights in the average being defined by the customer, and the averaged terms being values of the individual features of the product. These values are what makes up the product design. The paper presented a way of modelling the weights, which are otherwise usually unknown, so that companies could adopt changes to their product features and make them more competitive. Such a model of weights reflects a specific behaviour of the customer, and can be used in instances when companies believe that this behaviour could be in place. These instances include situations when the customer's evaluation of two products is very similar. This happens, for example, when a company begins to leave the stage of saturation in its business cycle. The approach of modelling the weights was compared to another approach that perhaps crosses one's mind in a more straightforward way, and can be thus considered more natural. The paper has shown, however, that the opposite will often be true, and the alternative approach will often be unsuitable for such modelling purposes.

At the very end of the analysis it is also necessary to note that customer satisfaction depends not only on the product itself, but also on pre-production and post-production services for which alternative ways of improvements would probably be more appropriate, given the qualitative rather than quantitative character of the services. Such alternative procedures include, for instance, techniques derived from Taguchi methods [13], [14].

\section{Appendix}

We shall prove the statement: if $a_{i}, i=1,2, \ldots, k$, $k>1$, are positive numbers, the symmetric matrix (1) is positive definite, and thus has an inverse, as well. To show the validity of this statement, we use induction. For $k=2$ and $k=3$, the corresponding determinants of (1) are positive, and the statement for such $k$ follows from the Sylvester's criterion [4]. Let us assume the statement holds for every integer from 2 up to an integer $k=n-1, n \geq 4$, and let us show that if this is the case, the statement also holds for the subsequent integer $k=n$, i.e. the matrix

$$
A=\left(\begin{array}{cccc}
a_{1}+a_{n} & a_{n} & \cdots & a_{n} \\
a_{n} & a_{2}+a_{n} & \cdots & a_{n} \\
\cdots & \cdots & \cdots & \cdots \\
a_{n} & a_{n} & \cdots & a_{n-1}+a_{n}
\end{array}\right)
$$


is positive definite. Subtracting the second-tolast row of $A$ from the last row of $A$ implies

$$
\begin{aligned}
& \operatorname{det} A=\left|\begin{array}{cccc}
a_{1}+a_{n} & a_{n} & \cdots & a_{n} \\
a_{n} & a_{2}+a_{n} & \cdots & a_{n} \\
\cdots & \cdots & \cdots & \cdots \\
a_{n} & a_{n} & \cdots & a_{n-1}+a_{n}
\end{array}\right|= \\
& =\left|\begin{array}{ccccc}
a_{1}+a_{n} & a_{n} & \cdots & \cdots & a_{n} \\
a_{n} & a_{2}+a_{n} & \cdots & \cdots & a_{n} \\
\cdots & \cdots & \cdots & \cdots & \cdots \\
0 & 0 & \cdots & -a_{n-2} & a_{n-1}
\end{array}\right|= \\
& =\underbrace{a_{n-1} \cdot(-1)^{2 n-2} \cdot\left|\begin{array}{cccc}
a_{1}+a_{n} & a_{n} & \cdots & a_{n} \\
a_{n} & a_{2}+a_{n} & \cdots & a_{n} \\
\cdots & \cdots & \cdots & \cdots \\
a_{n} & a_{n} & \cdots & a_{n-2}+a_{n}
\end{array}\right|}_{D_{1}}+ \\
& +\underbrace{\left(-a_{n-2}\right) \cdot(-1)^{2 n-3} \cdot\left|\begin{array}{ccccc}
a_{1}+a_{n} & a_{n} & \cdots & \cdots & a_{n} \\
a_{n} & a_{2}+a_{n} & \cdots & \cdots & a_{n} \\
\cdots & \cdots & \cdots & \cdots & \cdots \\
a_{n} & a_{n} & \cdots & a_{n-3}+a_{n} & a_{n} \\
a_{n} & a_{n} & \cdots & \cdots & a_{n}
\end{array}\right|}_{D_{2}}=
\end{aligned}
$$$$
=D_{1}+D_{2} \text {. }
$$

The term $D_{1}$ is positive, since $a_{n-1}$ is positive by assumption, $2 n-2$ is even for any integer $n$, and the determinant appearing in $D_{1}$ is positive by the induction assumption. As for the term $D_{2}$, subtracting the second-to-last row of the matrix appearing in $D_{2}$ from its last row, we have

$$
\begin{aligned}
& \left|\begin{array}{ccccc}
a_{1}+a_{n} & a_{n} & \cdots & \cdots & a_{n} \\
a_{n} & a_{2}+a_{n} & \cdots & \cdots & a_{n} \\
\cdots & \cdots & \cdots & \cdots & \cdots \\
a_{n} & a_{n} & \cdots & a_{n-3}+a_{n} & a_{n} \\
a_{n} & a_{n} & \cdots & \cdots & a_{n}
\end{array}\right|= \\
& =\left|\begin{array}{ccccc}
a_{1}+a_{n} & a_{n} & \cdots & \cdots & a_{n} \\
a_{n} & a_{2}+a_{n} & \cdots & \cdots & a_{n} \\
\cdots & \cdots & \cdots & \cdots & \cdots \\
a_{n} & a_{n} & \cdots & a_{n-3}+a_{n} & a_{n} \\
0 & 0 & \cdots & -a_{n-3} & 0
\end{array}\right|= \\
& =-a_{n-3} \cdot(-1)^{2 n-5} \cdot\left|\begin{array}{ccccc}
a_{1}+a_{n} & a_{n} & \cdots & \cdots & a_{n} \\
a_{n} & a_{2}+a_{n} & \cdots & \cdots & a_{n} \\
\cdots & \cdots & \cdots & \cdots & \cdots \\
a_{n} & a_{n} & \cdots & a_{n-4}+a_{n} & a_{n} \\
a_{n} & a_{n} & \cdots & \cdots & a_{n}
\end{array}\right| \text {. }
\end{aligned}
$$

Repeating this procedure, we see that

$$
\begin{aligned}
& D_{2}=\underbrace{\left(-a_{2}\right) \cdot\left(-a_{3}\right) \cdot \ldots \cdot\left(-a_{n-2}\right)}_{n-3 \text { terms }} \cdot \underbrace{(-1)^{5} \cdot(-1)^{7} \cdot \ldots \cdot(-1)^{2 n-3}}_{n-3 \text { terms }} . \\
& \cdot\left|\begin{array}{cc}
a_{1}+a_{n} & a_{n} \\
a_{n} & a_{n}
\end{array}\right| . \\
& D_{2}=(-1)^{n-3} \cdot\left(\prod_{i=2}^{n-2} a_{n-i}\right) \cdot(-1)^{5+7+9+\ldots+(2 n-3)} \cdot a_{1} \cdot a_{n} .
\end{aligned}
$$

The sign of $D_{2}$ depends only on the expression $(-1)^{n-3} \cdot(-1)^{5+7+9+\ldots+(2 n-3)}$. If $n$ is even, $n>5$, $(-1)^{n-3} \cdot(-1)^{5+7+9+\ldots+(2 n-3)}=(-1)^{n-3} \cdot(-1)^{5+(n+2)(n-4)}>0$, since $(n-3)+5+(n+2) \cdot(n-4)$ is even. If $n$ is odd, $n \geq 5$,

$$
(-1)^{n-3} \cdot(-1)^{5+7+9+\ldots+(2 n-3)}=(-1)^{n-3} \cdot(-1)^{(n+1)(n-3)}>0,
$$

since $(n-3)+(n+1) \cdot(n-3)$ is even again. The determinant of $A$ is therefore positive in either case, implying that $A$ is positive definite by the Sylvester's criterion.

\section{References}

[1] BAZARAA, M. S., SHERAL, H. D., SHETTY, C. M. Nonlinear Programming: Theory and Algorithms. 3rd ed. Hoboken: John Wiley and Sons, 2006. ISBN 978-0-471-48600-0. doi:10.1002/0471787779.

[2] CHOO, E. U., WEDLEY, W. C. Optimal Criterion Weights in Repetitive Multicriteria Decision-Making. Journal of Operational Research Society. 1985, Vol. 36, Iss. 11, pp. 983992. ISSN 0160-5682. doi:10.2307/2582430.

[3] CHOO, E. U., SCHONER, B. and WEDLEY, W. C. Interpretation of criteria weights in multicriteria decision making. Computer \& Industrial Engineering. 1999, Vol. 37, Iss. 3, pp. 527-541. ISSN 0360-8352. doi:10.1016/S03608352(00)00019-X.

[4] HORN, R. A., JOHNSON, C. R. Matrix Analysis. 1st ed. Cambridge: Cambridge University Press, 1985. ISBN 0-521-30586-1. doi:10.1017/CBO9780511810817.

[5] ISHIZAKA, A., NEMERY, P. Multicriteria Decision Analysis: Methods and Software. 1st ed. Chichester: John Wiley and Sons, 2013. ISBN 978-1-119-97407-9. doi: $10.1002 / 9781118644898$. 
[6] LUO, Y. B., YE, J. and MA, X. W. Multicriteria fuzzy decision-making method based on weighted correlation coefficients under intervalvalued intuitionistic fuzzy environment. In: IEEE 10th International Conference on ComputerAided Industrial Design \& Conceptual Design. Wenzhou (China), 2009. Vol. 3, pp. 2057-2060.

[7] MA, J., FAN, Z., HUANG, L. Subjective and Objective Integrated Approach to Determine Attribute Weights. European Journal of Operational Research. 1999, Vol. 112, Iss. 2, pp. 397-404. ISSN 0377-2217. doi:10.1016/S0377-2217(98)00141-6. [8] NUTT, P. C. Comparing methods for weighting decision criteria. OMEGA. 1980, Vol. 8, Iss. 2, pp. 163-172. ISSN 0305-0483. doi:10.1016/0305-0483(80)90020-1.

[9] O'SEARCOID, M. Metric Spaces. London: Springer-Verlag, 2007. ISBN 978-1-84628-369-7. [10] PARK, D. G., KWUN, Y. C., PARK, J. H., PARK, I. Y. Correlation coefficient of interval-valued intuitionistic fuzzy sets and its application to multiple attribute group decision-making problems. Mathematical and Computer Modelling. 2009, Vol. 50, Iss. 9-10, pp. 1279-1293. ISSN 0895-7177. doi:10.1016/j. mcm.2009.06.010.

[11] PEKELMAN, D., SEN, S. Mathematical programming models for determination of attribute weights. Management Science. 1974, Vol. 20, Iss. 8, pp. 1217-1229. ISSN 0025-1909. doi:10.1287/mnsc.20.8.1217.

[12] SOLYMOSI, T., DOMBI, J. A method for determining the weights of criteria: the centralized weights. European Journal of
Operational Research. 1986, Vol. 26, Iss. 1, p. 35-41. ISSN 0377-2217. doi:10.1016/03772217(86)90157-8.

[13] TEREK, M. Taguchiho prístup k inženierstvu kvality. In: Zborník Štatistické riadenie kvality z 9. Školy štatistiky Ekomstat 95. Trenčianské Teplice, 1995.

[14] TEREK, M., HRNČIAROVÁ, L. Taguchiho stratégia využitia experimentov $\mathrm{v}$ zlepšování kvality. In: Zborník z 9. medzinárodnej vedeckej konference Kvantitatívne metódy v ekonomii. Kompatibilita metodológie a praxe s podmienkami Európskej unie. Bratislava: FHI EU, 2003.

[15] WANG, Z., LI, K. W. and WANG, W. An approach to multiattribute decision making with interval-valued intuitionistic fuzzy assessments and incomplete weights. Information Sciences. 2009 , Vol. 179 , Iss. 17 , pp. 3026-3040. ISSN 0020-0255. doi:10.1016/j.ins.2009.05.001.

[16] YE, J. Multicriteria fuzzy decision-making method using entropy weights-based correlation coefficients of interval-valued intuitionistic fuzzy sets. Applied Mathematical Modelling. 2010, Vol. 34, Iss. 12, pp. 3864-3870. ISSN 0307904X. doi:10.1016/j.apm.2010.03.025.

Ing. Filip Tošenovský, Ph.D.

Silesian University

Faculty of Business Administration in Karviná

Department of Mathematical Methods

in Economics tosenovskyfilip@opf.slu.cz 


\section{Abstract}

\section{MULTICRITERIA DECISION-MAKING WEIGHTS AND A COMPETITIVE PRODUCT DESIGN}

\section{Filip Tošenovský}

The paper presents a general way of improving product features so that products were more competitive. The presented concept first represents the general standard of a product to be improved by a weighted average of the product feature levels, thus drawing on concepts from multicriteria decision-making theories. The paper then models the weights appearing in the average so that the average could be calculated, and the product could be improved, achieving at the same time that the modelled weights reflect a specific behaviour of the customer who is the ultimate judge of the product quality or standard. The reason for doing so is the fact that it is the customer who defines the weights which attach importance to each product feature. Since the behaviour of the customer is unknown, the weights are unknown, and must be modelled. If the weights are modelled in such a way that the resulting model reflects a specific customer behaviour, companies may refer to this model, when improving their products, provided there are reasons to believe that such a specific customer behaviour is occurring in the market. The approach to modelling the weights presented in the paper is compared to another approach for finding the weights, the alternative approach offering itself as a more natural way for these purposes. The paper shows, however, that the alternative approach lacks some desirable properties that the approach proposed in the paper is able to provide. The concepts presented in the paper are proved generally, using known mathematical optimization techniques, and the proved theory is accompanied by examples which support the validity of the general statements.

Key Words: Product evaluation, weighted average, weights, optimization, weight interpretation, cost-effective product improvement.

JEL Classification: C02, C61, L15, L21, M11, M21, O14, O32.

DOI: 10.15240/tul/001/2015-1-007 